\title{
EDITORIAL
}

\section{New technology, non-Western instruments and composition}

The theme of this issue allowed practitioners, many from an academic and 'art-music' perspective, to reflect on the process of combining digital technology and non-Western musical instruments in the creation of new works.

In the call for material for the issue, it was hoped to survey some of the traditional instruments being used, and views on the ways to incorporate them technically and sonically into new compositions. Questions were also raised about methods of performer/composer collaboration and the integration of live performers with contemporary sound production methods. Work on the computational modelling of non-Western instruments was also solicited, as was work in genres that used a hybrid of traditional instruments and new technology. There was also an aesthetic concern about the integration and juxtaposition of international and traditional approaches to music-making, and the artistic intentions and uses of non-Western instruments. Finally, submissions might cover something of the cultural and environmental influences on making works, and the level of engagement with local/traditional communities.

The topic is difficult to write about from a Western practitioner's perspective. Many Western 'art-music' composers have a limited understanding of the cultural context that non-Western musical instruments are drawn from, and increasing political correctness and/or cultural sensitivity regarding cultural appropriation has made the territory difficult for the uncommitted or simply curious. From another perspective, many fine non-Western instrumental practitioners and composers are not attached to 'art-music' or academic circles, or are not sympathetic to digital music idioms. Further, writing academic papers in English for them requires sacrificing time that could be spent creating music.

It is then rare to find composers or performers who are able to bridge these cultural and artistic divides, and who also have the financial support to allow exploration of the issues in written form.

The few intrepid souls from various parts of the globe included in this collection represent a small selection of work that deals with aspects of the central theme. In line with most Organised Sound articles, a common thread is that the authors give a background or context to work being undertaken, illustrate a process of engagement and/or invention, and apply the results to a creative output. It should be noted that most submissions have included sound and/or movie material that will be included with Vol. 10 No. 3.

In Matthew Burtner's 'Ecoacoustic and shamanic technologies for multimedia composition and performance', the author explores his connections with Alaskan culture, and how this has informed the creation of new multimedia instruments based on ritual objects in shamanic cultures. He also demonstrates how the processes used in his associated works are structurally tied to environmental systems in a technique he describes as 'ecoacoustics'.

Following this is Robert Gluck's 'eSaz: a nonWestern instrument in the context of a live electronic performance system'. Here, a classical Turkish lute fitted with electronic sensors and linked to a software interface designed in MAX/MSP is used as the basis for a performance system. The wide-ranging discussion includes instrument design, cross-cultural borrowing, and the how the author's Jewish identification influences some of the aesthetic choices made.

South American composer Rodolfo Coelho de Souza then examines the use of Brazilian folk instruments in his Concerto for Computer and Orchestra. The work uses original and transformed samples of the Berimbau, Cuica and Zunidores sounds, in combination with synthesised spectro-morphological variations of the sounds of these instruments. The author notes that his approach has implications both as a cultural signifier, and for the method of integrating the computer with the orchestra.

Two North Americans currently based in Hong Kong then present different approaches to the use of Chinese traditional instruments. Lydia Ayers describes her work on synthesising a collection of Chinese wind instruments using Csound. After describing the instruments and the method of synthesis, a summary of how they are rendered is described, and examples of their application cited from the author's works. In contrast, Christopher Keyes in 'Recent technology and the hybridisation of Western and Chinese musics' begins with an historical overview from a Western perspective. He argues that recent 
technologies have accelerated hybridisation but also solved many of the integral artistic problems. The outcome is also illustrated in a discussion of a selection of the author's electroacoustic works.

In the final paper dedicated to the theme, Ian Whalley looks at traditional New Zealand Mäori instruments, composition and digital technology. A main focus is on the collaborative work Hirini Melbourne and Richard Nunns undertook together and with others. It explores aesthetic, practical and cultural considerations integral to making music with the instruments using hybrid approaches.

Two other papers are included in the issue, in line with the Organised Sound policy of including 'off-theme' papers in each selection. In the first, Stefania Serafin and Juraj Kojs from Denmark look at computer models and compositional applications of plastic corrugated tubes. An application is illustrated in the composition Garden of the Dragon for amplified cellophane, plastic corrugated tubes, and electronics. The work was recently premiered at ICMC'04 in Miami, USA. Also from Denmark, Kristoffer Jensen in 'Atomic noise' discusses the use of unvoiced sounds considered to be of use in noise music. The research integrates different methods to add subtle variations and 'life' to noise. Methods of obtaining a harmonic sound from noise are also introduced, and the continuum from stochastic sound to "noisy harmonic sound' explored.

I hope this small collection will inspire others to explore the central theme through continuing work. I offer sincere thanks to Leigh Landy for his insight, good cheer and help in preparing this issue.

Ian Whalley

Department of Music University of Waikato 